\title{
L'évaluation de traitements et autres moyens de lutte contre le SIDA
}

Évaluer objectivement l'efficacité des traitements, voilà bien une nécessité que plus personne ne conteste, même si persistent çà et là d'importantes difficultés méthodologiques et, parfois, éthiques. Ces difficultés sont nettement amplifiées dans le cas du SIDA, maladie nouvelle, sévère, encore mystérieuse et remplie d'une charge affective et fantasmatique peu commune. L'appréciation de l'efficacité... voire de la non-nocivité des campagnes d'information à visée préventive reste encore plus difficile... d'autant plus que leur évaluation rigoureuse n'a guère été tentée ! Il est pourtant évident que c'est sur ces tentatives de « prévention primaire » que reposent essentiellement, pour l'instant, les espoirs de limitation du développement de l'épidémie actuelle.

\section{Daniel Schwartz Jean-Pierre Aboulker}

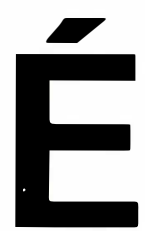

valuer rigoureusement des moyens de lutte contre le SIDA est une tâche ardue mais possible, à condition d'utiliser une méthodologie appropriée. Une telle méthodologie existe, elle a fait ses preuves abondamment pour l'évaluation des traitements dans de nombreuses maladies. On va en rappeler brièvement les principes, avant de montrer quelles difficultés elle rencontre dans l'application, soit à des traitements, soit à d'autres moyens de lutte, dans le cas du SIDA.

\section{L'évaluation des traitements}

Lorsqu'il s'est agi de démontrer le rôle de la streptomycine dans le traitement de la méningite tuberculeuse, jusque-là toujours mortelle, point n'était besoin de recourir à une technique sophistiquée. Mais de tels succès sont rares, et la thérapeutique progresse en règle générale par une succession de gains modestes que seule peut apprécier une évaluation rigoureuse. C'est une telle évaluation qu'a permise la méthode statistique, il y a quelque cinquante ans, aboutissant par une série de perfectionnements à une méthodologie maintenant très élaborée [1].
Les quatre phases. Dans le cas des médicaments, l'évaluation, après les indispensables études de laboratoire in vitro et sur animal, se déroule chez l'homme en quatre étapes. La phase l est l'étude de la tolérance (primum non nocere), la phase 2 vise à donner une première idée de l'efficacité, du métabolisme dans l'organisme, des meilleures modalités d'administration. La phase 3 vise à évaluer rigoureusement le nouveau traitement, à le situer dans l'arsenal des thérapeutiques existantes. La phase 4 est la surveillance du médicament après sa mise sur le marché, afin surtout de déceler d'éventuels effets indésirables, rares ou tardifs, que n'aurait pu mettre en évidence la phase 3 (Tableau I).

Dans le cas des traitements non médicamenteux (radiothérapie, chirurgie, etc.), l'individualisation de ces quatre phases est moins nette. C'est la phase 3, communément appelée «l'essai thérapeutique contrôlé », qui, dans tous les cas, constitue l'étape cruciale de l'évaluation d'un traitement. C'est elle qui va être plus particulièrement décrite.

L'essai thérapeutique contrôlé : quatre principes essentiels. 1. Un groupe recevant le traitement à évaluer est comparé à un groupe témoin 
soumis à un traitement classique, ou non traité s'il n'existe pas de traitement reconnu actif. Ce principe est parfois méconnu, un médecin qui a obtenu dans un groupe traité $40 \%$ de guérisons s'imagine qu'il a « évalué » le traitement. Comment pourrait-il en être ainsi, alors qu'il ignore quel pourcentage il aurait obtenu en l'absence d'un traitement ? Peut-être $40 \%$ aussi ? Peut-être même plus ? Ainsi, qui dit évaluation dit comparaison.

2. La comparaison des résultats dans les deux groupes doit, cela va de soi, faire appel à une méthodologie permettant de savoir si la différence observée peut résulter des seules fluctuations d'échantillonnage, ou est au contraire « significative». C'est le test statistique.

3. Une différence significative entre les groupes n'est pas nécessairement le fait des traitements. Une telle conclusion causale n'est possible que si les groupes sont, à part le traitement, strictement comparables. La constitution de groupes comparables pose un problème difficile. On ne peut considérer comme comparables les groupes de malades qui, au cours d'une époque donnée, « se trouvent » avoir reçu l'un ou l'autre des traitements à comparer; par exemple, pour un cancer, les malades irradiés et les malades opérés ; tout simplement parce que le cancérologue aura envoyé au chirurgien les meilleurs cas. On ne peut davantage considérer comme comparables les cas de deux époques différentes : indiquons, à titre d'exemple, que dans un grand hôpital parisien, à un moment donné, la mortalité pour un type de cancer a đû d'année en année ; l'explication était que la venue d'un chef de service réputé attirait des cas de plus en plus graves. Le défaut commun à ces exemples est que les groupes à comparer se constituent spontanément, en fonction d'une de leurs caractéristiques ou d'un événement, ce qui les rend - de ce fait non comparables. La difficulté est la même que celle rencontrée dans le domaine des sondages, lorqu'on cherche à obtenir un échantillon «représentatif ». On sait que la seule manière rigoureuse est le tirage au sort. Ici, de même, la seule manière rigoureuse d'obtenir des groupes comparables consiste à répartir les malades entre les deux groupes par une technique (la randomisation) utilisant le tirage au sort.

4. Des groupes comparables au départ peuvent perdre leur comparabilité en cours d'essai du seul fait de la connaissance du traitement reçu : d'abord au niveau des critères de jugement, la personne qui juge pouvant par partialité, même inconsciente, avantager le traitement qu'elle croit le meilleur; mais surtout au niveau de l'évolution même de la maladie, qui peut être influencée par des phénomènes de suggestion. D'autosuggestion d'abord : des études ont montré qu'un placebo, traitement inactif imitant dans sa présentation un traitement actif, pouvait, dans de nombreux cas, en reproduire les effets bénéfiques, et parfois les inconvénients secondaires. D'hétérosuggestion ensuite : on a constaté également que la foi du médecin dans le traitement peut influencer le cours de la maladie. On ne citera ici qu'un seul exemple, particulièrement spectaculaire ; quand parut sur le marché un «médicament miracle» contre l'asthme, un médecin décida de soumettre une de ses patientes à un essai rigoureux; il commanda au laboratoire, lors de crises successives, tantôt le médicament, tantôt du placebo. Il obtint régulièrement succès dans le premier cas, échec dans l'autre. Lorsqu'il rapporta ces résultats au laboratoire, il s'entendit répondre qu'on lui avait envoyé... à chaque fois du placebo ! La foi seule avait agi, illustrant de manière spectaculaire la relation « médecin-malade » [2].

Pour éliminer de tels artéfacts, on a imaginé des essais comportant l'ignorance du traitement reçu, au niveau du malade, ou même du malade et du médecin : l'essai en double insu ou double aveugle est celui où les traitements à comparer sont présentés sous une forme indiscernable, leur identité n'étant connue ni du malade ni du médecin. Ces deux traitements peuvent être le traitement à évaluer et un placebo qui l'imite.

Cette machinerie... Les principes précédents, rappelés dans le Tableau II sont essentiels pour une évaluation rigoureuse, mais la machinerie complexe à laquelle ils conduisent n'estelle pas irréaliste, inutile, inéthique?

- Irréaliste? Dans le seul domaine du cancer, on a dénombré, sur le plan international, pour la période 19701980 , près de 1000 essais avec tirage au sort.

- Inutile? Un seul exemple sera cité : en 1957, le vaccin Salk contre la poliomyélite a fait l'objet aux ÉtatsUnis d'un essai contrôlé sur près de 400000 enfants dont la moitié, déterminés par tirage au sort, reçurent un placebo [3]. La preuve de l'efficacité (et de l'innocuité) du vaccin fut ainsi obtenue en 15 mois, permettant sa diffusion très rapide. Quel contraste avec les innombrables controverses dont a fait l'objet le vaccin BCG, qui n'avait été soumis au départ à aucun essai de ce genre!

- Inéthique ? C'est, certes, l'impression qu'on ne peut manquer de ressentir devant le tirage au sort, ou l'essai en double insu. Cependant comme de nombreuses instances nationales ou internationales - le Comité Consultatif National d'Éthique pour les Sciences de la Vie et de la Santé a donné son aval à ces démarches, sous réserve que soient remplies certaines conditions [4].

Les essais thérapeutiques contrôlés doivent être utilisés pour évaluer des traitements soit curatifs (essais cliniques), soit préventifs (essais de prévention). Ces derniers présentent des difficultés particulières qui seront exposées à propos du SIDA.

\section{Les essais dans le domaine du SIDA}

Dans la lutte contre le SIDA, on peut prendre des mesures à trois niveaux, selon qu'on cherche à diminuer le risque (disons annuel), soit d'infection chez les sujets séronégatifs (prévention primaire), soit d'évolution vers le SIDA chez les sujets séropositifs (prévention secondaire), soit de décès chez les sujets atteints de SIDA (thérapeutique curative).

Les sujets séronégatifs, les séropositifs encore asymptomatiques - les «porteurs sains » - sont des cibles privilégiées pour lutter contre la maladie, parce qu'ils sont les plus nombreux et parce qu'il paraît toujours raisonnable d'agir le plus tôt possible; mais c'est là aussi que les difficultés sont les plus grandes pour les essais, du fait que les sujets sont sains ou apparemment sains. Aussi nous bornerons-nous à étudier les essais chez ces deux catégories de 
Tableau I

LES QUATRE PHASES CLINIQUES DE L'ÉVALUATION D'UN MÉDICAMENT

\begin{tabular}{|c|c|}
\hline Phase & Objectif \\
\hline $\begin{array}{l}1 \\
2 \\
3\end{array}$ & $\begin{array}{l}\text { Étude de la tolérance } \\
\text { Première idée de l'efficacité } \\
\text { Évaluation rigoureuse de l'efficacité (par comparaison avec un traite- } \\
\text { ment de référence) }\end{array}$ \\
\hline 4 & Uetection d etrets indesirables rares ou taraits. \\
\hline
\end{tabular}

sujets, en commençant par les porteurs sains.

Les essais chez les porteurs sains (prévention secondaire). Ils sont une foule - disons entre $200000 \mathrm{et}$ 400000 en France. Cliniquement sains, biologiquement atteints, méritent-ils leur nom ? S'agit-il vraiment de prévention? Mais peu importent les mots, le fait est que, pour une évaluation de traitements, la situation est la même que, par exemple, quand on vise à réduire le taux d'infarctus chez les sujets à haut risque, les difficultés seront donc celles de la prévention chez les sujets à risque. On va les exposer dans le cas général et montrer ensuite quelles complications supplémentaires interviennent dans le cas du SIDA.

l. Le sujet ne se sent pas malade, il n'est pas certain qu'il le deviendra. Faut-il le traiter ? Tout traitement a un coût, au sens large. Le bilan coûtavantages, toujours délicat pour un traitement nouveau, l'est bien davantage quand le sujet s'estime bien portant.

2. Malades ou pas malades, voilà en tout cas des sujets qui mènent une vie professionnelle et familiale normale, «hors les murs » de l'hôpital, privés de l'encadrement, de la surveillance, du lien étroit avec le per- sonnel soignant, qui sont en vigueur dans la plupart des essais à visée curative. Et le risque est grand que le traitement soit non pris, ou mal pris, ou abandonné, ou que le patient soit perdu de vue; d'où une source de biais importante.

3. Des sujets dits «à haut risque » ont certes un risque plus élevé que les autres, mais qui reste le plus souvent, en prévention, relativement faible. Un essai nécessitera alors, soit beaucoup de sujets, soit beaucoup d'années; en général, le risque est pratiquement le même d'une année à la suivante, de sorte que, par exemple, 1000 sujets suivis pendant cinq ans apportent la même information que 5000 sujets suivis pendant un an, on peut donc raisonner sur des sujets $\times$ années, la meilleure solution est alors de suivre beaucoup de sujets pendant peu de temps, car la durée est source de multiples complications (ne fût-ce qu'une information nouvelle, vraie ou fausse, sur l'efficacité du traitement étudié, voire l'apparition d'un nouveau traitement). Mais cette solution est d'une réalisation difficile et bien souvent l'essai devra durer longtemps, ce qui accroît les difficultés exposées au point 2.

4. Enfin, si l'on doit utiliser un pla- cebo, les difficultés vont grandir encore: va-t-on pouvoir maintenir dans l'essai pendant un temps long des patients informés que leur traitement est peut-être un placebo ?

Traiter pendant des années des centaines ou des milliers de patients apparemment non malades, «hors les murs », avec un traitement d'efficacité et de toxicité inconnues ou un placebo, avec un respect constant du protocole, voilà résumée la difficulté d'un « essai de prévention chez des sujets à haut risque ». Faut-il parler de difficulté ou d'impossibilité ? Le pari a été tenu, et quelques illustres succès parmi d'autres peuvent être cités: dans la seule prévention des maladies cardio-vasculaires, la $\mathrm{Hel}$ sinki Heart Study [5] a montré le rôle protecteur d'un fibrate sur l'incidence des coronaropathies dans un essai randomisé avec placebo et double aveugle portant sur 4801 hommes hypercholestérolémiques suivis pendant cinq ans; un essai américain portant sur 22000 hommes de plus de 40 ans, avec tirage au sort, placebo et double aveugle [6], a montré une forte diminution du taux d'infarctus dans le groupe «aspirine »; on a même été jusqu'à comparer l'effet de divers régimes alimentaires sur la cholestérolémie et le risque d'infarctus, chez des groupes numériquement importants déterminés par tirage au sort [7]. (Faut-il préciser que ces essais d'alimentation imposée... n'ont pas eu lieu en France ?). Possibles donc, de tels essais. Mais d'une difficulté qu'on imagine sans peine. Or, quand on passe au SIDA, la difficulté va être bien plus grande encore. Il suffit, pour s'en convaincre, de reprendre successivement les quatre points discutés ci-dessus.

1. La position du «porteur sain » vis-à-vis de sa maladie (ou de sa nonmaladie...) est plus qu'ambiguë, s'agissant d'une situation nouvelle, incomplètement connue. Pour une maladie qui a émergé il y a quelque sept ans, qui se développe lentement, comment savoir actuellement combien de porteurs sains y échapperont? Spontanément, ou grâce à l'apparition de traitements efficaces, - car le domaine, s'il est mal connu, fait l'objet d'un nombre incroyable d'études ? Le porteur sain peut donc penser qu'il ne sera jamais malade, et même n'avoir qu'une idée : oublier son état. 


\section{RÉFÉRENCES}

1. Schwartz D, Flamant R, Lellouch J. L'essai thérapeutique chez l'homme. Paris : Flammarion, $1981: 285$

2. Wolf S. Placebos : the effect of pharmacological agents on the nervous system. Proceedings of the association for research in nervous and mental disease. Baltimore Williams and Wilkins 1959: 37

3. Francis $T$, Korns RF, Voight RB, et al. Evaluation of 1954 field trials of poliomyelitis vaccine. Summary report. Am J Publ Health $1955 ; 45: 5$.

4. Comité Consultatif National d'Éthique pour les Sciences de la Vie et de la Santé. Problèmes d'éthique posés par les essais de nouveaux traitements chez l'homme. Rappor 1984. Paris: La Documentation Française, $1985: 1.43$.

5. Frick MH. Helsinki Heart Study : primary prevention trial with gemfibrozil in middle aged men with dyslipedemia. $N$ Engl J Med 317 : 1987 : 1237-45.

6. The Steering Committee of the Physicians Health study research group. Preliminary report : finding from the aspirin componen of the ongoing physicians' health study. $N$ Engl J Med 1988; 318 (4) : 262-4.

7. Richard JL. Les essais de prévention primaire multifactorielle des cardiopathies ischémiques. Rev Epidemiol Santé Publ $1985 ; 33: 121-33$

8. Fischl MA, Richman DD, Griéco Mh, et al. Efficacy of azidothymidine (AZT) in the treatment of patients with AIDS and AIDS related complex. $N$ Engl J Med 1987; 317 : 185-91.

9. Cambien J, Richard JL, Jacqueson A, Ducimetière $\mathrm{P}$. Analyse des résultats d'un essai où des groupes ont été randomisés. Rev Epidemiol Santé Publ 1981 ; 29 : 281-8.

10. Job-Spira N, Meyer L, Bouet E, Spira A Résultats de l'évaluation d'une campagne de prévention des maladies sexuellement transmissibles en France. Contr Fert Sex 1988; 16 (1) : 59-62

11. Schwartz D. L'irrésolu. In : Lellouch J, ed. Présent et Futur de l'Épidémiologie. Paris : INSERM, $1988 ; 3546$.

12. Ngugi EN, Simonsen JN, Bosiré M, et al. Prevention of transmission of human immunodeficiency virus in Africa : effectiveness of condom promotion and health education among prostitutes. Lancet 1988 ; 2 : 887-90.

13. IVth International Conference on Aids. Stockholm International Fairs. Stockholm :
Donc pas de traitement! D'autant que les traitements connus sont le plus souvent toxiques. D'un autre côté, le risque de SIDA chez un porteur sain est certainement élevé et, logiquement, plus on agit tôt plus l'effet est grand. Donc il faut le traiter! C'est dire que le bilan coûtavantages est singulièrement délicat. 2. Ces patients «hors les murs» vivent sous haute pression; parce que la maladie est non seulement nouvelle, mal connue, objet d'incessantes découvertes, mais aussi mythique, entourée de fantasmes, et qu'elle est la proie des médias pour le meilleur et pour le pire. Pour le meilleur : le SIDA est sans doute la seule maladie pour laquelle on puisse trouver l'information la plus complète, signée des plus gands noms, jusque dans les kiosques des gares. Et il n'est pas certain que ceci facilite le traitement ou l'essai d'un traitement... Surtout quand voisine le pire : des annonces fracassantes de molécules efficaces, sans preuve valable, se succèdent, et voilà que les patients saisis d'espoir changent de traitement, ou d'hôpital, et que l'essai est compromis. Versatilité bien plus grande encore si les patients sont des toxicomanes.

3. Le risque annuel de SIDA grandit au cours du temps, il est, pour les cinq années suivant la contamination, de l'ordre de $0 \%, 0 \%, 3 \%, 5 \%$ et $7 \%$. On ne peut plus alors raisonner sur des sujets $X$ années et rechercher un suivi court en augmentant le nombre de sujets : si l'on a affaire à des sujets à un stade précoce, il faut non seulement en étudier un très grand nombre, mais aussi les suivre très longtemps. Il y a à cela d'ailleurs, une autre raison. Ce qu'on demande ici aux médicaments, c'est essentiellement une prolongation de la survie. On ne peut se contenter de conclure à partir d'une amélioration de paramètres biologiques (antigénémie p 25, nombre de cellules T4...) plusieurs médicaments sont actuellement susceptibles d'entraîner de telles améliorations à court terme, sans qu'on puisse prévoir ni leur efficacité ni leur toxicité à long terme. Pour ces deux raisons, un essai sur plusieurs années s'impose. Ce qui multiplie les difficultés exposées au point 2 .

4. Enfin, le placebo est nécessaire.
D'abord pour les raisons habituelles nécessité de juger pareillement l'évolution du mal dans les groupes traité et témoin, possibilité d'un effet placebo - non que la suggestion puisse éviter le SIDA, mais on ne saurait exclure qu'elle le retarde. Ensuite, parce que l'évolution vers le SIDA dépend peut-être de facteurs comportementaux : des réinfections par voie sexuelle ou toxicomanie intraveineuse pourraient activer le processus. Or des sujets non traités se comporteront sans doute autrement que des sujets traités. Enfin, des sujets non traités risqueraient, bien plus que les traités, de disparaître à la première rumeur d'une nouvelle molécule efficace, créant un biais. Mais si le placebo est nécessaire, son acceptation pose des problèmes bien plus délicats que dans toute autre maladie.

Quand on voit toutes ces difficultés, on comprend aisément qu'un essai chez les porteurs sains est une vraie gageure. De nombreuses tentatives ont vu le jour, suscitées par l'énormité de l'enjeu ; mais de manière le plus souvent sporadique, avec des effectifs trop faibles ou des méthodes imparfaites, et elles sont restées sans lendemain. La molécule faisant l'objet à ce jour des essais les plus importants est l'azido-thymidine ou «AZT». Cet antiviral a fait ses preuves [8] chez des sujets atteints de SIDA ou de manifestations cliniques sévères de l'infection (ARC); la figure 1 indique, à titre d'exemple, la nette diminution de fréquence qu'il entraîne dans l'apparition des maladies opportunistes. Il est donc urgent d'évaluer si son utilisation chez des porteurs sains peut ralentir l'évolution vers le SIDA. Cette molécule est toxique, mais elle paraît mieux tolérée à ce stade que chez les malades atteints de SIDA. Sept essais avec diverses posologies et un groupe placebo sont en cours ou en projet de par le monde, totalisant près de 9000 patients à suivre pendant deux à trois ans. C'est là sans doute une évaluation d'une grande difficulté, mais qui est la plus indiquée dans l'état actuel de la question. Elle est généralement entourée de précautions exceptionnelles, telles que : surveillance continue de la tolérance, analyses au cours de l'essai (selon une méthodologie statistique appropriée) pour y mettre fin plus tôt que 


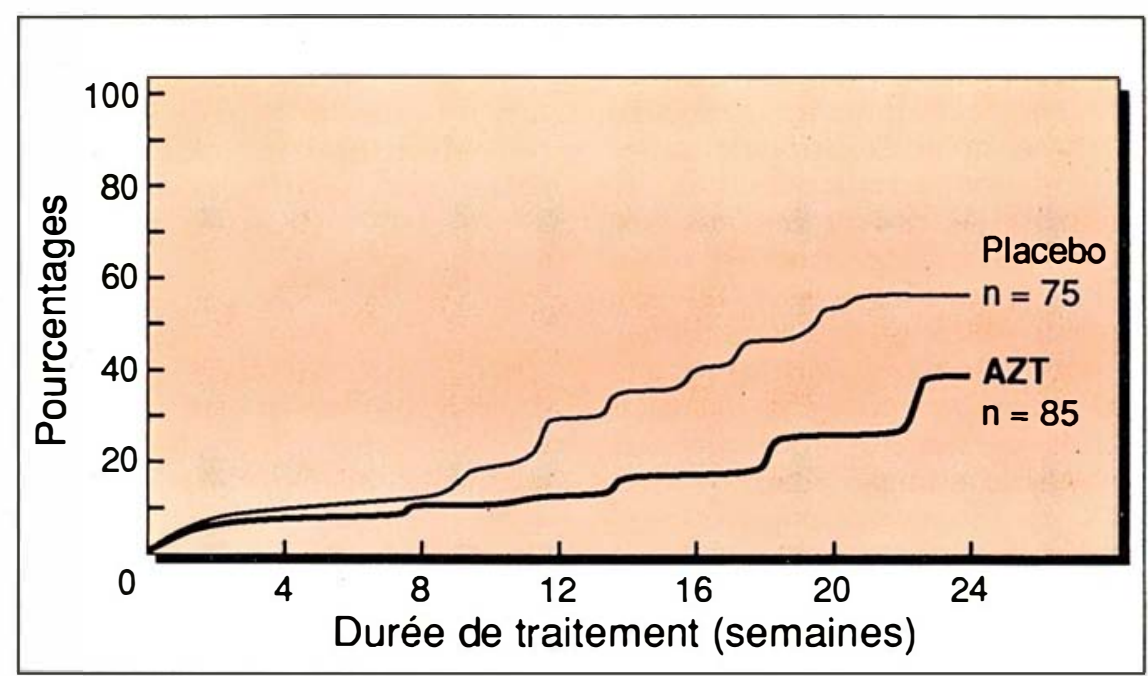

Figure 1. Pourcentage, on fonction du temps, de patients ayant développé des infections opportunistes, chez des malades atteints de SIDA recevant, soit de I'AZT, soit du placebo, dans l'étude [8] (avec l'autorisation du New England Journal of Medicine, juillet 1987, vol. 317, no 4, p. 188).

prévu si possible, constitution d'un groupe indépendant d'experts pouvant intervenir à tout moment sur son déroulement en fonction d'informations en provenance de l'essai ou de l'extérieur.

Sans doute convient-il de mentionner, après toutes les difficultés énoncées, un élément qui, en revanche, est favorable: des patients, plus nombreux qu'on ne pourrait le croire, consentent à participer activement à des essais, même avec placebo, mûs par un mouvement de solidarité dans la lutte contre un mal exceptionnel. Les essais chez les séronégatifs (la prévention primaire). Aussi longtemps qu'on manque de traitements efficaces, il faut viser plus en amont : avant la contamination. La règle d'or «agir sur le plus grand nombre et le plus tôt possible » conduit à une cible plus privilégiée encore que les porteurs sains: la population des séronégatifs (ou présumés tels). Cette prévention primaire peut être envisagée de deux manières: par la voie biologique (vaccination), ou par l'action sur les facteurs de risque (comportement sexuel, toxicomanie...).

1. La vaccination. Qu'elle concerne tous les séronégatifs ou seulement des séronégatifs à risque élevé, elle nécessitera une évaluation par les méthodes habituelles de l'essai thérapeutique, comme on l'a fait déjà pour de nombreux vaccins, un des $\mathrm{m} / \mathrm{s} n^{\circ} 3 \mathrm{vol} .5$, mars 89 gues, autorisation de la publicité sur les préservatifs). Pour évaluer de telles mesures, il faut surmonter deux difficultés principales, assez classiques en prévention primaire.

La première est le choix du critère d'efficacité. Ainsi une campagne anti-tabac en milieu scolaire devrait être évaluée par la diminution de l'incidence de certains cancers et des maladies cardio-vasculaires. Il faudrait attendre des années! Aussi se contente-t-on souvent de noter ce que les élèves auront retenu de la campagne. C'est très insuffisant: tel, convaincu des dangers du tabac à dix ans, sera peut-être grand fumeur à quatorze. On peut alors porter l'attention sur la diminution réelle de consommation de tabac. Bien qu'imparfaite, c'est là une approche acceptable. La situation est la même dans le cas du SIDA. Une campagne d'information en milieu scolaire devrait théoriquement être évaluée par la fréquence ultérieure des séroconversions. Voilà qui est difficilement concevable! On sera alors tenté de prendre seulement comme critère ce que les écoliers ont retenu du message, mais il serait beaucoup plus indiqué de connaître les modifications de comportement sexuel. Les interroger à ce sujet est imaginable - mais combien plus délicat que de les interroger sur leur consommation de tabac! On peut aussi utiliser des «marqueurs», comme l'incidence des maladies sexuellement transmissibles. Cette incidence est certes difficile à évaluer, mais moins que celle des séroconversions.

La seconde difficulté est d'ordre méthodologique. Il est usuel d'évaluer l'efficacité d'une mesure par la comparaison «avant-après ». On compare, par exemple, l'opinion d'écoliers sur les dangers du tabac avant et après la diffusion d'un message. L'apparente rigueur de ce procédé n'a d'égale que son évidente fausseté. Entre les moments «avant » et « après », bien des facteurs ont pu modifier le savoir des écoliers, ne fûtce que l'avalanche de tous autres messages reçus de l'entourage et des médias, et rien ne permet d'affirmer que la modification constatée est imputable au message à évaluer. Ici comme dans toute évaluation de traitement, il faut constituer des groupes traité et témoin par tirage au sort. 
Mais on rencontre alors une difficulté qui n'existe pas dans l'essai clinique, qui est l'impossibilité d'isoler des individus: comment faire pour que le professeur transmette un message à certains écoliers tirés au sort et pas à d'autres ? Et, en admettant même qu'il le fasse, comment éviter la «contagion » qui fera passer le message des uns aux autres? La solution existe : il faut prendre pour unités de tirage au sort, non des individus, mais des groupes: des classes ou, mieux des écoles dans l'exemple précédent. A titre d'exemple, citons une étude française visant à évaluer une intervention hygiénodiététique dans le domaine de la prévention cardio-vasculaire [9]. La population professionnelle concernée est divisée en services. Il a paru difficile de procéder à un tirage au sort des individus, conduisant à la présence quotidienne dans un même service de sujets dont certains, et pas d'autres, seraient soumis à une campagne intensive de prévention. On a donc préféré tirer au sort des services, de sorte que, sur 132 finalement retenus, 64 ont subi et 68 n'ont pas subi la campagne de prévention. De même, une campagne de prévention des maladies sexuellement transmissibles en France a été évaluée par comparaison de départements expérimentaux et témoins tirés au sort [10].

Une telle méthodologie peut, dans certains cas, être utilisée dans le domaine du SIDA. Des projets sont actuellement à l'étude pour évaluer une action d'éducation à l'école en constituant, à l'intérieur d'un département, deux groupes de secteurs tirés au sort.

Sans doute une approche de ce type est-elle parfois impossible (par exemple, pour évaluer un message télévisé à l'échelon national), et souvent très difficile, mais on ne devrait éliminer l'expérimentation avec tirage au sort qu'après s'être assuré qu'elle est réellement impossible. Et s'il en est ainsi, qu'on ne renonce pas pour autant à une approche relativement rigoureuse ; dans tout un domaine de l'épidémiologie, celui de la recherche étiologique, l'expérimentation avec tirage au sort est impossible; on ne peut évaluer le rôle du tabac, et moins encore celui de la cholestéro- tirage au sort. Les épidémiologistes ont alors mis au point un ensemble de méthodes et de critères permettant d'estimer qu'une relation de cause à effet est, sinon certaine, du moins très probable [11]. Parmi ces méthodes figure par exemple l'analyse multivariée: les fumeurs et les non fumeurs diffèrent par de nombreux facteurs (consommation de café, d'alcool, etc.), de sorte qu'on ne sait si l'excès de cancers bronchiques est imputable au tabac plutôt qu'à certains de ces facteurs ; l'analyse multivariée permet de comparer la fréquence du cancer chez les fumeurs et non fumeurs à égalité de tels facteurs. Un procédé de ce genre a été utilisé récemment pour comparer deux programmes d'éducation chez des prostituées, à partir de deux groupes constitués sans tirage au sort, mais en prenant en compte dans l'analyse les facteurs apparents de leur différence [12].

De tels soucis de rigueur sont malheureusement exceptionnels. Les Actes du dernier congrès international sur le SIDA [13] font apparaître un criant contraste entre la multitude des programmes de prévention et la médiocrité de leur évaluation. La multitude... et le coût élevé. On est confondu de constater que des opérations bien plus coûteuses qu'un essai thérapeutique n'exigent pas l'arsenal des même précautions. Alors que l'évaluation d'une thérapeutique demande quatre phases et le passage devant un comité d'éthique, les mesures de prévention ne paraissent pas obéir aux mêmes règles. Qu'en est-il, pour commencer, de la phase 1 de tolérance? Primum non nocere: un message télévisé est-il forcément anodin? «La parole est plus dangereuse que l'épée ", dit un proverbe italien. Des paroles, une image, peuvent faire bien plus de mal qu'un médicament, d'autant plus qu'elles frappent des millions de sujets, parmi lesquels ce qui n'est pas le cas des médicaments! - des sujets non concernés : de jeunes enfants, par exemple. Des essais préliminaires sont-ils toujours tentés sur différentes catégories de public, avec la rigueur voulue? Il est difficile de le savoir car, à la différence encore des essais thérapeutiques, les protocoles - s'ils existent - ne sont pas publiés. En fait, les responsables des programmes de prévention et ceux qui évaluent des traitements forment deux mondes séparés, alors que la méthodologie devrait bien souvent être la même.

\section{Conclusion}

«Il n'est pas conforme à l'éthique d'administrer un traitement dont on ne sait, alors qu'on pourrait le savoir, s'il est le meilleur des traitements disponibles; voire même s'il est efficace et s'il n'est pas nocif. L'évaluation d'un nouveau traitement est un devoir », proclame le Comité Consultatif National d'Éthique pour les Sciences de la Vie et de la Santé dans son avis sur les essais de nouveaux traitements [4], ajoutant que l'évaluation doit obéir à une méthodologie rigoureuse, comportant notamment le tirage au sort. Cette conclusion s'applique au SIDA comme à toute autre maladie. En l'état actuel, les traitements les plus rentables sont, soit les médicaments qui agiraient le plus tôt possible chez des séropositifs pour diminuer le risque de SIDA, soit mieux encore, des mesures visant plus en amont à diminuer le risque d'infection.

L'évaluation de ces médicaments ou de ces mesures soulève une série de difficultés dont aucune n'est spécifique du SIDA, mais qui dans le SIDA sont simultanément présentes et avec une particulière gravité.

Dans l'évaluation des médicaments, la difficulté est grande parce qu'il faut mener des essais avec suivi de longue durée sur de nombreux sujets apparemment sains, informés qu'une moitié d'entre eux reçoivent un placebo, et qui vivent «hors les murs » de l'hôpital dans un climat passionnel qu'exacerbent les rumeurs.

Dans l'évaluation des mesures préventives, les obstacles sont d'un autre ordre, Des difficultés matérielles ıendent souvent impossible l'expérimentation avec tirage au sort. Mais celle-ci est praticable plus souvent qu'on ne le pense et, lorsqu'elle ne l'est pas; on dispose quand même de méthodes relativement satisfaisantes. Tant que les mesures de prévention primiaire resteront la grande priorité, l'effort principal devrait porter sur leur évaluation rigoureuse 


\section{Summary}

Evaluation of treatments and other means of fighting AIDS

A rigorous methodology is now available to compare treatments. The major step is the controlled clinical trial. Two randomized groups are compared : a « treated » group receives the treatment to be assessed and a "control » group receives a « reference » treatment, or no treatment if none exists. It is often advisable that the nature of treatment not be known by the patient, and sometimes even by his doctor. This is a double blind controlled trial. Should the controls not be treated, they receive an inert placebo undistinguishable from the treatment. In the fight against AIDS, the most efficient programmes are the ones directed towards infected but still asymptomatic patients (secondary prevention) or towards uninfected subjects (primary prevention by information campaigns, legislation, etc.). The assessment of such programmes by the controlled clinical trials methodology meets serious difficulties, which always exist in prevention but are amplifield in the case of AIDS. In secondary prevention, the difficulty lies in the follow-up of thousands of apparently healthy subjects, informed that their treatment might be a placebo without them knowing it, and living outside the hospital in an environment aggravated by rumors. In primary prevention, such as in information campaigns, random assignment of subjects is often impossible. Random assignment of groups (school class for instance) is seldom feasible. When randomization is impossible, alternative methods, which are satisfactory although less rigorous, are available. As long as primary prevention remains the priority, every effort must be made towards as strict as possible assessment of programmes proposed in that field.

\section{TIRÉS A PART}

D. Schwartz.

$\mathrm{m} / \mathrm{s} n^{\circ} 3$ vol. 5, mars 89 